\title{
Micromechanical Modelling of the Superthermoelastic Behavior of Materials Undergoing Thermoelastic Phase Transition
}

\author{
D. Entemeyer, E. Patoor, A. Eberhardt and M. Berveiller
}

Laboratoire de Physique et Mécanique des Matériaux, URA 1215 du CNRS, Institut Supérieur de Génie Mécanique et Productique, Ile du Saulcy, 57045 Metz, France

\begin{abstract}
The superelasticity of the Shape Memory Alloys (SMA) is well known nowadays, but few models describe the non isothermal behavior of SMA.

In this paper, we have developped a micromechanical modelling of the SMA's thermomechanical behavior based on a kinematical description of the strain mechanisms. In order to describe the microstructure evolution of the material, we define a thermodynamical potential in which volume fractions of the different variants of martensite used as the internal variables. This study allow us to determine the constitutive equations for the local behavior. Global one is then obtained using a scale transition method; the self-consistent one. This model is able to predict not only the macroscopic behavior but also the evolution of the variants of martensite in the material. The results are in good agreement with experimental data and observations.
\end{abstract}

\section{INTRODUCTION}

The behavior of the SMA is mainly due to the phase transition which occurs during different thermomechanical loadings. The formation and the growth of the different variants of martensite are the physical mechanisms responsible of the global behavior.

Many models are developped to predict the SMA's behavior under a mechanical loading (superelasticity), using a phenomenological like for instance [1] [2] [3] or a micromechanical approach [4] [5]. In most cases, they are not able to predict the non isothermal behavior of SMA, called in this paper: superthermoelasticity.

Superthermoelasticity is observed during temperature changes with applied stress. It's well known that temperature changes only create self-accommodating variants of martensite and don't produce any macroscopical deformations. At the contrary, applied stress during cooling privileges some variants and thus we observe a macroscopical strain due to the oriented martensite. This overall total strain increases with the applied stress until a certain saturation value. Some phenomenological modellings take into account this phenomenon [6]. They consider two types of martensite (the self-accommodating martensite and the oriented one) each having its own volume fraction although there's no crystallographical difference between these two types of martensite. Kinematical evolution of these volume fractions is difficult to determine.

The micromechanical modelling we present in this paper is able to determine the single crystal flow rule using the same assumptions than in the case of superelasticity. The polycrystalline behavior is obtained using the self-consistent homogeneization method. A new integral equation is determined to take into account the temperature effect. The results of these theoretical analyses have been compared with experimental curves and are in good agreement.

\section{SINGLE CRYSTAL BEHAVIOR}

This behavior has been described in previous paper [5] for superelasticity. No additional assumptions are needed at this level to account superthermoelastic behavior. Major points of this approach are recalled in the following.

We considere that no plasticity occurs during the phase transition. At a point $r$ in the material, the total strain field $\varepsilon(r)$ is defined as the sum of the elastic, thermal and transformation strain. 
Transformation strain $\varepsilon^{n}$ linked to the formation of a variant $n$ is defined from the habit plane $\mathbf{n}^{\mathbf{n}}$, the direction of transformation $\mathrm{m}^{\mathrm{n}}$ and the amplitude $\mathrm{g}$ of the transformation in this previous direction.

$$
\forall n, \quad \varepsilon_{i j}^{n}=\frac{1}{2} g\left(n_{i}^{n} m_{j}^{n}+m_{i}^{n} n_{j}^{n}\right)=g R_{i j}^{n}
$$

Moreover, we assume that the elastic modulus $C$ and the thermal expansion factor $\alpha$ are homogeneous, isotropical and identical in both phases. Thus, we write the macroscopic deformations:

$$
E_{i j}=E_{i j}^{e}+E_{i j}^{t h}+E_{i j}^{T} \quad \text { with: } \quad E_{i j}^{T}=\sum_{n} \varepsilon_{i j}^{n} f^{n}
$$

$\mathrm{f}^{\mathrm{n}}$ is the volume fraction of the variant $\mathrm{n}$. Some physical constraints are exerted on these variables:

$$
\forall n \quad f^{n} \geq 0 \quad \text { and } \quad \sum_{n} f^{n}=f \leq 1
$$

The evolution of the total strain is obtained from a thermodynamical potential function of the control parameters (applied stress $\Sigma$ and temperature $T$ ) and the set of internal variables $f^{n}$. In an unitary volume undergoing a thermoelastic transformation, the complementary free energy $\psi$ is constitued by chemical energy $\Delta G_{c h}$, elastic energy $W_{e l}$ and by interfacial energy $W_{S}$.

$$
\psi\left(\Sigma_{\mathrm{ij}}, \mathrm{T}, \mathrm{f}^{\mathrm{n}}\right)=-\left(\Delta \mathrm{G}_{\mathrm{ch}}+\mathrm{W}_{\mathrm{el}}+\mathrm{W}_{\mathrm{S}}-\Sigma_{\mathrm{ij}} \mathrm{E}_{\mathrm{ij}}^{\mathrm{T}}\right)
$$

Chemical free energy is commonly considered to be a linear function of the temperature. The oblate shape of the martensite plates leads to assume that interfacial energy is negligible in regard of the elastic one. Elastic energy is divided in two parts due to incompatibilities in the transformation strain field. Micromechanical analyses [7] have shown that elastic energy associated with these incompatibilities can be represented by an interaction matrix $\mathrm{H}^{\mathrm{nm}}$ that includes the shape and the orientation factors.

Finally, the complementary free energy associated with the transformation in an unitary volume of crystal is expressed as [5]:

$$
\psi\left(\Sigma_{\mathrm{ij}}, \mathrm{T}, \mathrm{f}^{\mathrm{n}}\right)=-\mathrm{B}\left(\mathrm{T}-\mathrm{T}_{0}\right) \sum_{\mathrm{n}} \mathrm{f}^{\mathrm{n}}-\frac{1}{2} \Sigma_{\mathrm{ij}} \mathrm{C}_{\mathrm{ijk} \mathrm{k}}^{-1} \Sigma_{\mathrm{kl}}+\Sigma_{\mathrm{ij}} \sum_{\mathrm{n}} \varepsilon_{\mathrm{ij}}^{\mathrm{n}} \mathrm{f}^{\mathrm{n}}-\mathrm{g}^{2} \sum_{\mathrm{n}, \mathrm{m}} \mathrm{H}^{\mathrm{nm}} \mathrm{f}^{\mathrm{n}} \mathrm{f}^{\mathrm{m}}
$$

where $T_{0}$ denotes the thermodynamical equilibrium transformation temperature.

Thermodynamical force $F^{n}$ acting on a variant $n$ is obtained from the classical partial derivation of this potential respected to $\mathrm{f}^{\mathbf{n}}$. Existence of the kinematical constraints (3) imposes to define a constrained potential $\mathrm{L}\left(\Sigma_{\mathrm{ij}}, \mathrm{T}, \mathrm{f}^{\mathrm{n}}\right)$ using the Lagrange multipliers $\lambda_{\mathrm{i}}$. We obtain for driving force:

$$
\mathrm{F}^{\mathrm{n}}=\Sigma_{\mathrm{ij}} \varepsilon_{\mathrm{ij}}^{\mathrm{n}}-\mathrm{B}\left(\mathrm{T}-\mathrm{T}_{0}\right)-\mathrm{g}^{2} \sum_{\mathrm{m}} \mathrm{H}^{\mathrm{nm}} \mathrm{f}^{\mathrm{m}}-\lambda_{0}+\lambda_{\mathrm{n}}
$$

In order to take into account the hysteretic effect, a pseudo-dissipative potential $W_{d}$ is introduced [5]. Second law of the thermodynamics and the energy balance require:

$$
\left.\dot{\psi}\right|_{\Sigma, T}=\dot{\mathrm{W}}_{\mathrm{d}}=\sum_{\mathbf{n}} \mathrm{F}^{\mathrm{n}_{\mathbf{f}}} \dot{\mathrm{n}}^{\mathrm{n}} \geq 0
$$

We assume the formation and the growth of the different variants of martensite happens when the driving force $F^{n}$ reaches a critical value $F_{c}$ (a material positive constant). From expression (6), each variant has to observe local transformation criteria, defined in the forward and reverse case:

$$
\Sigma_{\mathrm{ij}} \varepsilon_{\mathrm{ij}}^{\mathrm{n}}-\mathrm{B}\left(\mathrm{T}-\mathrm{T}_{0}\right)-\mathrm{g}^{2} \sum_{\mathrm{m}} \mathrm{H}^{\mathrm{nm}_{\mathrm{f}} \mathrm{m}}-\lambda_{0}+\lambda_{\mathrm{n}}= \pm \mathrm{F}_{\mathrm{c}}
$$

Kinematical conditions (3) impose to verify $n+1$ additional conditions [5]:

From this analysis, at a point $\mathrm{r}$ in the material, the stress field is linked to the strain field and the temperature by two thermomechanical modulus $l(r)$ et $m(r)$ :

$$
\dot{\sigma}_{\mathrm{ij}}(r)=\mathrm{l}_{\mathrm{ijk}}(r) \dot{\varepsilon}_{\mathrm{kl}}(r)-\mathrm{m}_{\mathrm{ij}}(\mathrm{r}) \dot{\mathrm{T}}
$$


We obtain:

$$
\left\{\begin{array}{c}
1_{i j k l}(r)=C_{i j k l}-C_{i j k l} \sum_{n} R_{i j}^{n} \sum_{m}\left[H^{n m}+R_{i j}^{n} C_{i j k l} R_{k l}^{m}\right]^{-1} R_{i j}^{n} C_{i j k l} \\
m_{i j}(r)=C_{i j k l} \alpha_{i k l}-C_{i j k l} \sum_{n} R_{i j}^{n} \sum_{m}\left[H^{n m}+R_{i j}^{n} C_{i j k l} R_{k l}^{m}\right]^{-1}\left[(B / g)+R_{i j}^{n} C_{i j k l} \alpha_{k l}\right]
\end{array}\right.
$$

Microscopical properties are then entirely determined for the single crystal. In the polycrystalline case, in which the structure is granular, the local loading condition is unknown due to the internal stress field. This difficulty can be solved by a homogeneization method. We used the self-consistent one. Until now, applications are made considering the temperature rate $\dot{T}$ equal to zero. Take into account the temperature effect needs to improve the scale transition method used.

\section{POLYCRYSTALLINE BEHAVIOR}

The non isothermal character of the loading conditions is account in this part. A more complete thermomechanical integral equation is defined.

\subsection{Global behavior and homogeneization}

Polycrystalline behavior is defined in the same manner of the single crystal case (9):

$$
\dot{\Sigma}_{\mathrm{ij}}=\mathrm{L}_{\mathrm{ijkl}}^{\text {eff }} \dot{\mathrm{E}}_{\mathrm{kl}}-\mathrm{M}_{\mathrm{ij}}^{\text {eff }} \dot{\mathrm{T}}
$$

These quantities are linked to the local ones by four globalisation tensors $A(r), B(r), a(r)$ and $b(r)$.

$$
\begin{aligned}
& \dot{\varepsilon}_{i j}(r)=A_{i j k l}(r) \dot{E}_{k l}+a_{i j}(r) \dot{T} \\
& \dot{\sigma}_{i j}(r)=B_{i j k l}(r) \dot{\Sigma}_{k l}+b_{i j}(r) \dot{T}
\end{aligned}
$$

Combination of equations (9), (11) and (12) leads to the following results:

$$
\begin{gathered}
L_{i j k l}^{e f f}=\frac{1}{V} \int_{V} l_{i j k l}(r) A_{k l m n}(r) d V \\
M_{i j}^{e f f}=\frac{-1}{V} \int_{V}\left(l_{i j k l}(r) a_{k l}(r)-m_{i j}(r)\right) d V
\end{gathered}
$$

The globalisation tensors are calculated from a thermomechanical integral equation. This one links the local strain field up to the imposed kinematical conditions.

\subsection{Thermomechanical integral equation}

First assumption is to consider a homogeneous reference medium characterized by a set of uniform tangent modulus ( $\mathrm{L}^{\text {eff }}$ and $\mathrm{M}^{\text {eff }}$ ) and subjected to an uniform strain $\mathrm{E}^{0}$. We consider:

$$
l_{i j k l}(r)=L_{i j k l}^{e f f}+\delta l_{i j k l}(r) \quad \text { and } \quad m_{i j}(r)=M_{i j}^{\text {eff }}+\delta m_{i j}(r)
$$

At the micro level, the local behavior (9) may be introduced in the equilibrium condition. According to assumptions (14) and with respect to the continuity of deformation, we can write:

$$
\mathrm{L}_{\mathrm{ijkl}}^{\mathrm{eff}} \dot{\mathrm{u}}_{\mathrm{k}, \mathrm{lj}}+\left[\delta \mathrm{l}_{\mathrm{ijkl}}(\mathrm{r}) \dot{\varepsilon}_{\mathrm{kl}}-\delta \mathrm{m}_{\mathrm{ij}}(\mathrm{r}) \dot{\mathrm{T}}\right]_{, \mathrm{j}}=0
$$

In this equation, $(\delta \mathrm{I}(r) \dot{\varepsilon}-\delta \mathrm{m}(\mathrm{r}) \dot{\mathrm{T}})_{, j}=\mathrm{f}_{\mathrm{i}}$ is corresponding at some fictitious volumic forces. The equation (15) can be transformed in an integral equation using the Green tensor method. Indeed, the displacement rate field may be written with the Green tensor definition:

$$
\dot{\mathrm{u}}_{\mathrm{n}}(\mathrm{r})=\int_{\mathrm{V}} \delta_{\mathrm{nl}} \delta\left(r-r^{\prime}\right) \dot{\mathrm{u}}_{1}\left(r^{\prime}\right) d V=-\int_{V} L_{\mathrm{ijkl}}^{\text {eff }} G_{\mathrm{jn}, \mathrm{ik}}\left(r-r^{\prime}\right) \dot{\mathrm{u}}_{1}\left(\mathrm{r}^{\prime}\right) d V
$$


Integrating by parts and using the Ostrogradsky theorem, the equation (16) can be divided in two parts: the first is the solution of the fictitious homogeneous medium $\left(\dot{u}_{m}^{0}\right)$ and the second is due to the microheterogeneous properties of the real medium.

$$
\dot{u}_{m}(r)=\dot{u}_{m}^{0}+\int_{V^{\prime}} G_{m i, j}\left(r-r^{\prime}\right)\left[\delta l_{i j k l}\left(r^{\prime}\right) \dot{\varepsilon}_{k l}\left(r^{\prime}\right)-\delta m_{i j}\left(r^{\prime}\right) \dot{T}\right] d V^{\prime}
$$

After derivation and symetrisation, we obtain:

$$
\dot{\varepsilon}_{m n}(r)=\dot{E}_{m n}^{0}+\int_{V^{\prime}} \Gamma_{m n i j}\left(r-r^{\prime}\right)\left[\delta l_{i j k l}\left(r^{\prime}\right) \dot{\varepsilon}_{k I}\left(r^{\prime}\right)-\delta m_{i j}\left(r^{\prime}\right) \dot{T}\right] d V^{\prime}
$$

This relation constitues an integral equation binding the local strain rate $\dot{\varepsilon}(\mathrm{r})$ and the thermomechanical loading characterized by $\dot{\mathrm{E}}^{0}$ and $\dot{\mathrm{T}}$. Its solution allows to calculate the globalisation tensors $\mathrm{A}(\mathrm{r})$ and $\mathrm{a}(\mathrm{r})$. To solve such an equation, the self-consistent method is used.

\subsection{One site self consistent approximation}

This approximation assumes the polycrystalline to be constitued by $\mathrm{N}$ grains $\mathrm{I}$ having volume $\mathrm{V}^{\mathrm{I}}$ and volume fraction $\mathrm{f}^{\mathrm{I}}$. In each grain the behavior is regarded as uniform, i.e.:

$$
X(r)=\sum_{I=1}^{N} X^{I} Y^{I}(r) \quad \text { with } \quad\left\{\begin{array}{c}
X=\sigma_{i j} \text { or } \varepsilon_{i j} \text { or } \delta l_{i j k l} \text { or... } \\
Y^{I}(r)=\left\{\begin{array}{l}
0 \text { if } \mathrm{r} \notin V^{I} \\
1 \text { if } \mathrm{r} \in V^{I}
\end{array}\right.
\end{array}\right.
$$

According to these assumptions (18) becomes:

$$
\dot{\varepsilon}_{\mathrm{ij}}^{\mathrm{I}}=\dot{\mathrm{E}}_{\mathrm{ij}}^{\mathrm{O}}+\sum_{\mathrm{J}=1}^{\mathrm{N}} \mathrm{T}_{\mathrm{ijkl}}^{\mathrm{IJ}}\left[\delta \mathrm{l}_{\mathrm{klmn}}^{\mathrm{J}} \dot{\varepsilon}_{\mathrm{mn}}^{\mathrm{J}}-\delta \mathrm{m}_{\mathrm{kl}}^{\mathrm{J}} \dot{\mathrm{T}}\right]
$$

with: $T_{\mathrm{ijkl}}^{\mathrm{LJ}}=\frac{1}{\mathrm{~V}^{\mathrm{I}}} \int_{\mathrm{V}^{\mathrm{I}}} \int_{\mathrm{V}^{\mathrm{J}}} \Gamma_{\mathrm{ijkl}}\left(\mathrm{r}-\mathrm{r}^{\prime}\right) \mathrm{dV}^{\mathrm{I}} \mathrm{dV^{ \textrm {J } }}$

Replacing all the grains $\mathrm{J} \neq \mathrm{I}$ by the equivalent homogeneous medium, we obtain:

$$
\dot{\varepsilon}_{\mathrm{ij}}^{\mathrm{I}}=\left[\mathrm{I}_{\mathrm{ijmn}}-\mathrm{T}_{\mathrm{ijkl}}^{\mathrm{II}} \delta \mathrm{I}_{\mathrm{klmn}}^{\mathrm{I}}\right]^{-1} \dot{\mathrm{E}}_{\mathrm{mn}}-\left[\mathrm{I}_{\mathrm{ijmn}}-\mathrm{T}_{\mathrm{j} \mathrm{j} \mathrm{l} l}^{\mathrm{II}} \delta \mathrm{l}_{\mathrm{klmn}}^{\mathrm{I}}\right]^{-1} \mathrm{~T}_{\mathrm{mnpq}}^{\mathrm{II}} \delta \mathrm{m}_{\mathrm{pq}}^{\mathrm{I}} \dot{\mathrm{T}}
$$

Identifying with equation (12) leads to the following results:

$$
\left\{\begin{array}{c}
A_{\mathrm{ijkl}}^{I}=\left[I_{\mathrm{ijkl}}-T_{\mathrm{ijmn}}^{\mathrm{II}} \delta 1_{\mathrm{mnkl}}^{\mathrm{I}}\right]^{-1} \\
\mathrm{a}_{\mathrm{ijj}}^{\mathrm{I}}=-\left[\mathrm{I}_{\mathrm{ijkl}}-\mathrm{T}_{\mathrm{ijmn}}^{\mathrm{II}} \delta \mathrm{I}_{\mathrm{mnkl}}^{\mathrm{I}}\right]^{-1} \mathrm{~T}_{\mathrm{klpq}}^{\mathrm{II}} \delta \mathrm{m}_{\mathrm{pq}}^{\mathrm{I}}
\end{array}\right.
$$

Equations (23), (13) and (11) define the complete global behavior from local properties $\varepsilon(r), \sigma(r), \delta l(r)$ and $\delta \mathrm{m}(\mathrm{r})$. Themselves are determined from the microstructure evolution of the different grains that are constituing the polycrystalline with the equations (10) and (9).

\section{RESULTS}

Numerical results are applied to $\mathrm{Cu}-\mathrm{Zn}$-Al Shape Memory Alloy. In these alloys the habit plane normal $\mathbf{n}$ and the transformation direction $m$ of the variants are $\left[\begin{array}{lll}2 & 11 & 12\end{array}\right]$ type. The variants observed in these alloys are forming six self-accommodating groups each of them constitued by four variants. Transformation displacement $g$ is about 0.23 [8]. Coefficient $B$ is equal to $0.2 \mathrm{MPa} \cdot \mathrm{K}^{-1}$.

Data related to the material are the shear modulus $\mu=30 \mathrm{GPa}$, the Poisson ratio $v=0.3$ and the thermal 
expansion factor $\alpha=15.10^{-6} \mathrm{~K}^{-1}$. Interaction matrix $\mathrm{H}^{\mathrm{nm}}$ is constitued with two types of terms: a weak one $\left(\mathrm{H}_{1}=\mu / 2000\right)$ for self-accommodating variants and a strong one $\left(\mathrm{H}_{2}=\mu / 100\right)$ for the other. The polycrystalline structure is described by 100 grains with an orientation randomly chosen for each one. Results obtained in that way are compared with experimental tests performed by Vacher [9] and Bourbon [10]. Successfully, we compare a superelastic tensile test (figure 1) and a thermal cycling with constant applied stress equal to $65 \mathrm{MPa}$ (figure 2). Experimental tests [9] [10] showed that the maximal macroscopic transformation deformation obtained for a complete transformation (100\% of martensite) increases with the applied stress until a certain saturation value. Experimental points in figure 3 show this phenomenon and give the numerical results obtained with our model. In the numerical case, the phenomenon is less visible than in the case of experience but the general tendancy is obtained.

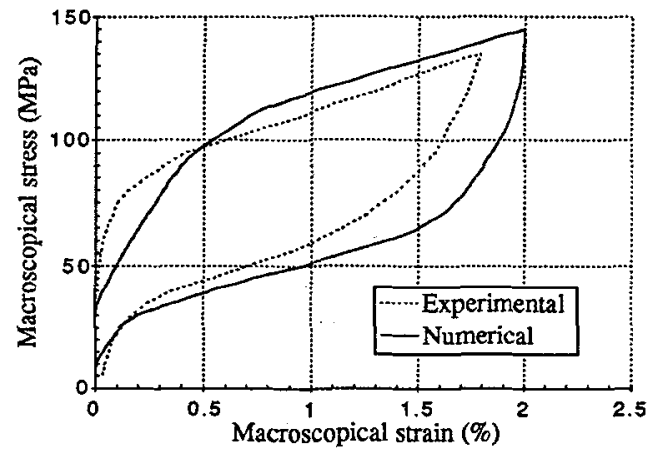

Figure 1: Tensile test in superelasticity at $T=329 \mathrm{~K}$. Comparison between experimental results [9] and self-consistent modelling ( $\mathrm{Ms}=313 \mathrm{~K}, \mathrm{Af}=325 \mathrm{~K}$ ).

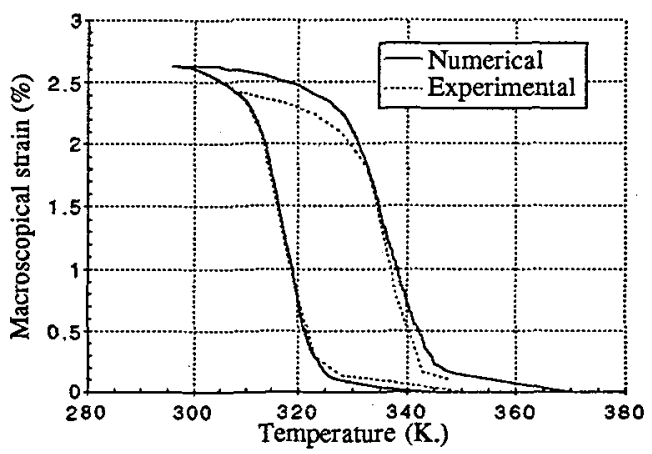

Figure 2: Thermal cycling with a 65MPa applied stress. Comparison between experimental results [10] and selfconsistent modelling (Ms $=313 \mathrm{~K}, \mathrm{Af}=325 \mathrm{~K})$.

Such results are obtained without any additionnal assumptions because the model takes into account the fact that in a polycrystalline material the stress field is not constant through the material due to the interactions between grains. Thus, in each grain, the stress field is divided with the macroscopic stress field (the same in all the grains) and an internal stress field which varies in the medium. Each of the grains constituing the polycrystalline material is individually and successively treated during the numerical process, it is supposed to be surrounded by an equivalent homogeneous medium and the choice of the activated variants is based on an energy minimisation. So, all grains have their own behavior and the microstructural changes can be observed in each grain. Figures 4, 5 and 6 show the evolution of the volumic fractions in the same grain for three different loading conditions.

For a superelastic tensile test (figure 4), the first variant formed is the best oriented (variant V9) in that case. But this variant has a large growing only at the very begining of the transformation. At further stage variants V21 and V24 from the same self-accommodating group (not too badly oriented) increase. Such phenomena is directly related to the internal stress field due to strain heterogeneities. During a thermal cycling with high applied stress (figure 5), the behavior obtained is quite similar. However, orientation of the variants is less important than in the case of superelasticity, so the badly oriented variant V12 appears at the beginning of the transformation due to its belonging to the same self-accommodating group than the variant V9. Indeed, we know that temperature changes only create self-accommodated martensite. Looking at the figure 6, we can see that lower is the applied stress, more important is the number of variants formed during the transformation. Moreover, variants 21,22 and 24 is included in the same selfaccommodating group and constitue more than $90 \%$ of the martensite at the end of the transformation. The coexistance of these 3 variants strongly reduced the contribution of this grain to the macroscopic transformation strain. That is in fact the physical reason of the behavior shown in figure 3 . A tentative explanation of the discrepancy between numerical results and experimental one at low stress level can be derived from these evolution. In fact the interaction matrix used in the local behavior (10) is only valid when few variants are formed. 


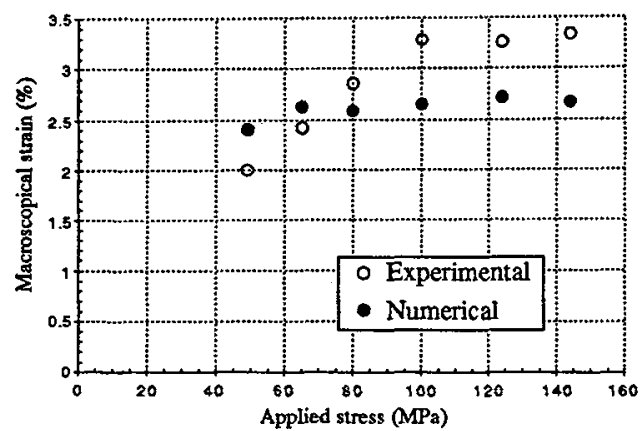

Figure 3: Effect of the constant applied stress on the maximum transformation strain during the first cooling. Comparison between experimental results [10] and self-consistent approximation ( $\mathrm{Ms}=313 \mathrm{~K}$ ).

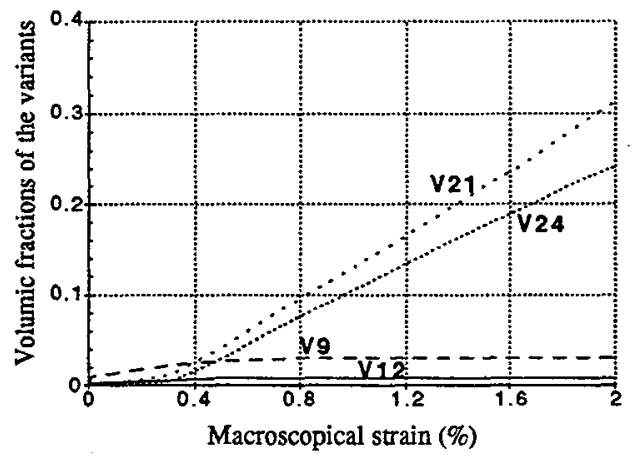

Figure 5: Evolution of variants during a thermal cycling with applied stress equal to $144 \mathrm{MPa}$.

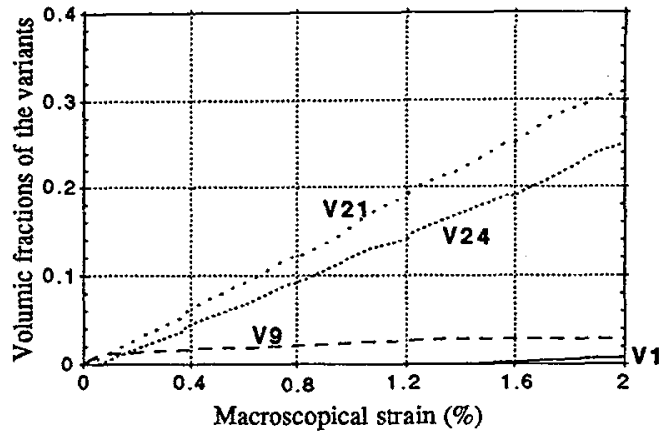

Figure 4: Evolution of variants during a tensile test.

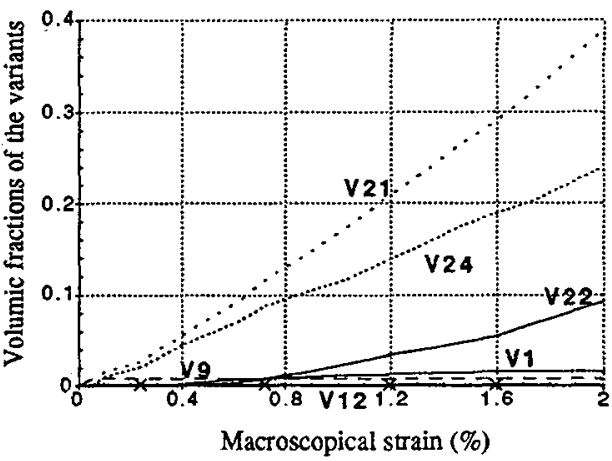

Figure 6: Evolution of variants during a thermal cycling with applied stress equal to $49 \mathrm{MPa}$.

\section{CONCLUSION}

Numerical results obtained from the micromechanical approach are in good agreement with experimental results. In addition, the model is in agreement with experimental observations about the microstructure evolution. Such results for anisothermal loading are obtained without any additionnal assumption because we choose the variant of martensite to be the description scale in order to take into account microstructural aspects and then, to link the global behavior up to the physical parameters responsible of the deformation.

\section{BIBLIOGRAPHIE}

[1] Raniecki B., Lexcellent C. and Tanaka K., Arch. Mech., 3 (1992) pp.261-284.

[2] Brinson L.C., J. of Intell. Mater. Syst. and Struct., Vol.4 (1993) pp.229-242.

[3] Boyd J.G., D.C. Lagoudas, Chicago, AMD-Vol. 189 / PVD-Vol. 292 (1994) pp.159-177.

[4] Sun Q.P. and Hwang K.C., J. Mech. Phys. Solids, 41 (1993) pp.1-6.

[5] Patoor E., Eberhardt A., Berveiller M., Procs ASME WAM'94, Chicago, I L (U.S.A.), AMD-Vol. 189 / PVD-Vol. 292 (1994) pp.23-37.

[6] Leclercq S., Bourbon G. and Lexcellent C., Procs ESOMAT' 94, Barcelona, Les Editions de Physique, France (1994) pp.513-518.

[7] Bensalah M.O., Thèse d'Etat, Université Mohammed V, Rabat (1992) Maroc.

[8] De Vos J., Aernoudt E. and Delaey L., Z. Metallkde, 69 (1978) pp.438-444.

[9] Vacher P. and Lexcellent C., Procs ICM 91, Kyoto, Pergamon Press (1991) pp.231-236

[10] Bourbon G., Lexcellent C., Leclercq S., Procs ICOMAT 95, Lausanne, Les Editions de Physique, France (1995) This conference. 